\title{
The 'intrinsic' system in the human cortex and self-projection: a data driven analysis
}

\author{
Shahar Arzy ${ }^{\mathrm{a}, \mathrm{d}}$, Yossi Arzouan ${ }^{\mathrm{b}}$, Esther Adi-Japha ${ }^{\mathrm{c}}$, Sorin Solomon ${ }^{\mathrm{b}}$ \\ and Olaf Blanke ${ }^{\mathrm{d}}$
}

\begin{abstract}
Information received by the human cortex is supplied by two main sources: extrinsic stimuli delivered by the external environment and intrinsic information regarding the body and self. We reanalyzed electrophysiological data involving the same external stimuli, but manipulating the degree of 'self-projection' to locations inside and outside the body border. Electrical neuroimaging and spatial principal component analysis (PCA) showed a bipartition of the cerebral cortex into two main subsystems: occipital and frontal activity was similar across tasks; activity in temporo-parietal and anterior frontal regions was modulated according to the manipulation of self-projection in a given task. These data suggest that the first system relates to external stimulus processing ('extrinsic') and the latter one relates to processing of the 'internal milieu' of
\end{abstract}

\author{
body and self ('intrinsic') . NeuroReport 21:569-574 \\ (c) 2010 Wolters Kluwer Health | Lippincott Williams \& \\ Wilkins.
}

NeuroReport 2010, 21:569-574

Keywords: default network, internal milieu, intrinsic system, self-projection

${ }^{a}$ Department of Neurology, Hadassah Hebrew University Hospital, ${ }^{b}$ Racah Institute of Physics, Hebrew University, Jerusalem, 'The School of Education, Bar-llan University, Ramat-Gan, Israel and 'Laboratory of Cognitive Neuroscience, Brain Mind Institute, Ecole Polytechnique Fédérale de Lausanne (EPFL), Lausanne, Switzerland

Correspondence to Shahar Arzy, MD, PhD, Department of Neurology, Hadassah Hebrew University Hospital Jerusalem, Israel

Tel: +972 2 6777741; fax: +972 2 6437782; e-mail: shahar.arzy@hcuge.ch

Received 16 February 2010 accepted 15 March 2010

\section{Introduction}

The cerebral cortex receives two main types of input: extrinsic information, delivered as various external stimuli through the different senses, and intrinsic information, concerning the internal environment and bodily states [1]. Although increases in brain metabolism in response to the extrinsic input as measured with PET are rarely greater than $5-10 \%$ of the resting metabolism of the brain, much larger amounts of energy are consumed by the brain to maintain its intrinsic activity [2]. Assuming that the intrinsic activity is prominent while no external stimuli are presented, a number of functional neuroimaging studies found a network of brain regions that were activated during the resting state, but were deactivated (showing signal reduction) after presentations of external stimuli [2-5]. This 'resting state' or 'default' network is composed mainly of regions in the posterior parietal, superior temporal, and medial prefrontal cortex showing correlated activity in the absence of external stimuli, suggesting an intrinsic influence on this system [6]. Another network composed of the frontal and occipital regions, which have been associated with processes commonly recruited by cognitive paradigms, was found to be deactivated during resting state [6,7]. Similarly, using functional MRI with correlation and clustering analyses, Golland et al. discovered a bipartition of the brain into two systems: an extrinsic (fronto-occipital)

\footnotetext{
Supplemental digital content is available for this article. Direct URL citations appear in the printed text and are provided in the HTML and PDF versions of this article on the journal's Website (www.neuroreport.com).
}

system, related to external stimuli, and an intrinsic (temporo-parietal) system, related to the intrinsic information processing, which overlaps with the abovementioned resting state associated network $[8,9]$.

When investigating the 'resting state', however, the above-mentioned studies did not directly scrutinize the 'intrinsic' processes, but rather the absence of 'extrinsic' ones. More direct evidence might come from the investigation of processing related to one's own body and self, 'intrinsic' components that were shown to activate the core regions in the intrinsic system (for review see Ref. [10]). Such processing has recently been related to the newly introduced concept of 'selfprojection' [11]. 'Self-projection' has been defined as 'a shift of perception from the immediate environment to the alternative, imagined environment' [1,11]. Similarly, one might experience oneself to be localized within the spatial limits of one's own body or alternatively to 'project' oneself to another 'location' outside the limits of one's own body. This 'self-projection' may enable us to experience ourselves and the environment from various spatial locations and perspectives, which might represent possible past and future spatial locations of ourselves. In earlier studies, we were able to identify brain mechanisms related to such spatial 'self-projection' and localized them predominantly to the temporo-parietal cortex $[12,13]$. Moreover, we showed that interference with these mechanisms might lead to illusory own body perceptions such as 'out-of-body experiences' (during which one experiences one's self to be located outside one's body and 
to see from this location one's 'physical' body) or 'feeling-ofa-presence' of an illusory person close by (which we showed to be a 'self-projection' to the peripersonal space) $[14,15]$. Here, we hypothesized that tasks with different levels of 'self-projection' will be more strongly associated with brain activity in the intrinsic than in the extrinsic system. To this aim, we used behavioral tasks involving the same external stimuli but different levels of 'self-projection', as recorded earlier $[12,13]$. In an in-depth analysis we here applied electrical neuroimaging and spatial principal component analysis (sPCA) on electrophysiological data, collected from two of our earlier studies [12,13], and looked for the main spatial components of the data and their relationships to the different tasks.

\section{Methods}

This study is based on a new conception and reanalysis of data collected from earlier studies $[12,13]$. Only a brief description of the paradigm is provided here; for a full description, refer to the original studies. These studies included three behavioral tasks. In a so-called own-body transformation task (OBT-task) participants were asked to 'project' themselves to the location and orientation of a schematic human figure. Either the right or the left hand of the figure was marked, and the participants indicated as to which one, as if they were taking the figure's location. In a 'mirror' task (MIR-task), the same figure was shown, but the participants were instructed to imagine that it was their mirror reflection, as seen from their habitual self-location. Finally, in a lateralization task (LAT-task), the participants were shown the same figures and asked to indicate whether the marked hand was in the right or in the left side of the screen, with no self projection. All three tasks involved the same visual stimuli, requirements, and response mode. However, in the OBT-task, the participants had to mentally 'project' themselves to another self-location; in the MIR-task they processed their mirror image without changing their habitual self-location; and in the LAT-task, judgements were made from the habitual self-location with respect to an external reference frame with no 'self-projection'. As was reported earlier [12,13], different behavioral patterns were found for the three different tasks. The participants were faster for the LAT-task than for the OBT-task and for the OBT-task than for the MIR-task (Fig. 1b; all $P<0.001$ ). Similar patterns were found for the error rates (Fig. 1b).

\section{Functional localization}

Continuous electroencephalogram was acquired and analyzed as described earlier [12,13]. On the basis of the results from accumulating evidence of functional neuroimaging, we clustered the original areas following Brodmann's classification into 18 'functional Brodmann Areas' (fBAs), adapted to the spatial resolution of electrical neuroimaging and reflecting their functional role as measured by functional neuroimaging (Supplemental Table S1,
Supplemental Digital content 1, http://links.lww.com/WNR/ A71) [16]. Each fBA is supposed to serve as a different modality in the brain hierarchy [16]. To analyze the recorded brain activity in the spatial domain, the average evoked potentials for each condition and participant were subjected to a low-resolution brain electromagnetic tomography (LORETA) analysis [17]. LORETA's 2394 voxels were transformed into fBA labeling with each $\mathrm{fBA}$ comprising approximately 150 voxels, and then subjected to a sPCA analysis. PCA is a multivariate technique to determine 'data-driven' component measures in which data are ordered in the sequence of activation, using either time series (temporal PCA, tPCA) or brain regions (sPCA) as the variable. The basic assumption of PCA is that mixed signals can be decomposed into a linear combination of principal component coefficients (factor loadings) and associated weights (factor scores). The decomposition procedure results in component coefficients that are orthogonal to each other and are thought to highlight patterns that emerge from the overall variance, whereas the factor scores represent the relative contribution of each pattern (for details and references see Supplementary Methods online http://links.lwwe.com/WNR/A50). In a second step, the sPCA differences as identified in the group-averaged data were verified statistically in the sPCA components of the individual participants using a two-tailed $t$-test. This allows to determine the time-windows of significant differences $(P<0.05)$ between the two components across participants. Statistical comparisons were made on the sPCA of the individual participants for each sPCA component in each hemisphere, in both the experiments.

\section{Results}

sPCA analysis of the electrical neuroimaging data comparing the OBT-task with the LAT-task (experiment 1) and OBT-task with the MIR-task (experiment 2) yielded the same two PCA components for each of the three tasks in both the comparisons for both hemispheres (Fig. 2a) with eigen values greater than $1(11.4,2.8)$, explaining $96 \%$ of the variance. In both the experiments 1 and 2, the first component was composed of the brain regions in the lateral parietal and temporal cortex as well as a prefrontal region (fBAs 5,7-11,17; Fig. 2b), and the second component was composed of the frontal and occipital regions (fBAs 1,2,3,14,18; Fig. 2b). The corresponding activities for each task in each hemisphere were reconstructed from the sPCA scores (Fig. 3). Similar results were found in both experiments: the first sPCA component (sPCA1) was significantly more prominent than the second component (sPCA2) in the period after approximately $250 \mathrm{~ms}$, peaking at approximately $400 \mathrm{~ms}$, and sPCA2 was more prominent than sPCA1 from stimulus onset to approximately $250 \mathrm{~ms}$, peaking at approximately $100 \mathrm{~ms}$.

Next, we compared each sPCA component separately for each task and hemisphere in experiment 1 (OBT vs. LAT) and experiment 2 (OBT vs. MIR). In both 
(a)
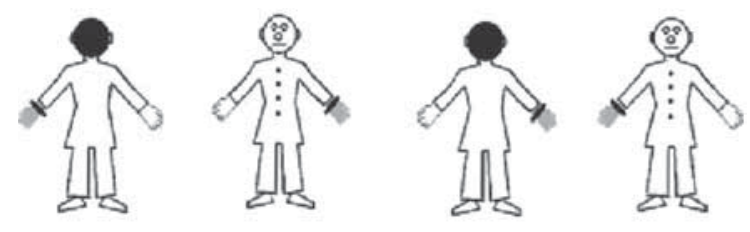

\begin{tabular}{|l|c|c|c|c|}
\hline OBT-task & left & left & right & right \\
\hline MIR-task & right & right & left & left \\
\hline LAT-task & left & right & right & left \\
\hline
\end{tabular}

(b)
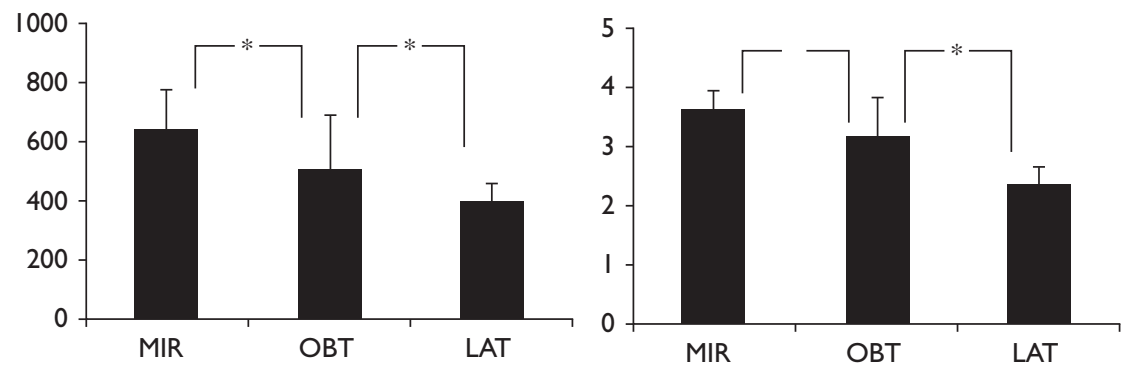

Task and behavior. (a) The four different stimuli as used in the own-body transformation (OBT), mirror (MIR), and lateralization (LAT) tasks are shown. Correct responses are indicated below each figure for each task. (b) Reaction times (ms) and error rates (\%) in the OBT, MIR, and LAT tasks. *Significant differences.

experiments, sPCA1 was modulated by the task, whereas sPCA2 showed only minimal differences among the tasks. In experiment 1 , sPCA1 was significantly stronger in the OBT-task than in the LAT-task mainly in the periods from approximately 200 to $400 \mathrm{~ms}$ and from approximately 550 to $700 \mathrm{~ms} \quad(P<0.05)$. This was found bilaterally without significant interhemispheric differences (Fig. 3b, upper row). In experiment 2, sPCA1 was higher in the OBT-task than in the MIR-task in the period from approximately 250 to $600 \mathrm{~ms}$. The right hemisphere showed higher activity than the left hemisphere, especially for the OBT-task (Fig. 3a, upper row). Conversely, in both experiments 1 and 2, sPCA2 showed only minimal differences between the tasks and between the hemispheres (Fig. 3a and b, lower rows). Comparison of experiments 1 and 2 showed higher activity of sPCA1 for the OBT-task than for the MIR-task and LAT-task, at around $300-600 \mathrm{~ms}$.

Taken together, across all tasks and comparisons, sPCA analysis showed two distinct components: the first component (sPCA1), comprised mainly of a system of brain regions in the lateral parietal and temporal cortex (fBAs 5,7-11,17), was more prominent after approximately $250 \mathrm{~ms}$ and showed different activities between tasks. The second component (sPCA2) comprised mostly the frontal and occipital regions (fBAs $1,2,3,14,18$ ), was more prominent until approximately $250 \mathrm{~ms}$ and showed similar activities across all tasks. We therefore associate the first component to 'intrinsic' activity, as it was only this component that was changing according to the level of 'self-projection', and the second component to perception and processing of 'extrinsic' stimuli, compatible with their anatomical distribution, as discussed below.

\section{Discussion}

The decomposition of electrical brain activity into two main systems that can be related, one to intrinsic information processing and the other to external stimuli, in this study, is parallel to the findings of earlier functional MRI studies in resting state and visual perception $[3,4,6$, $8,9,18]$. In addition to these earlier findings, in this study, activations of brain regions comprised in the first 'intrinsic' system $[8,9]$ were dependent on the level of 'self-projection' (OBT > MIR > LAT), whereas activation in the 'extrinsic' system remained stable across all tasks. In the following paragraphs, the functional and anatomical bases of the intrinsic and extrinsic systems are discussed, including the brain regions, laterality, and timing of activation.

Intrinsic and extrinsic brain regions in the frontal cortex The frontal regions are traditionally considered to encode complex cognitive functions, such as planning and executive control and high-order self-related and self-initiated functions [1,19]. Our analysis found a subdivision of the frontal cortex into intrinsic (prefrontal and frontal pole; fBA 17) and extrinsic (inferior frontal and prefrontal 
Fig. 2

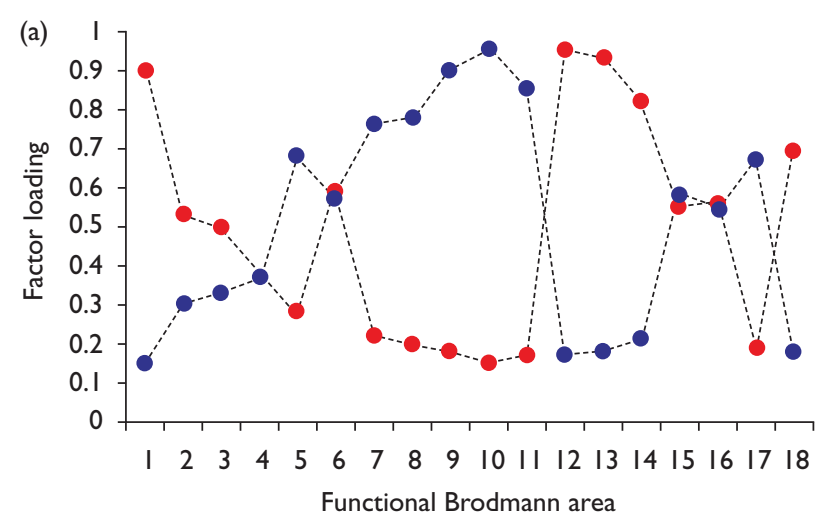

(b)

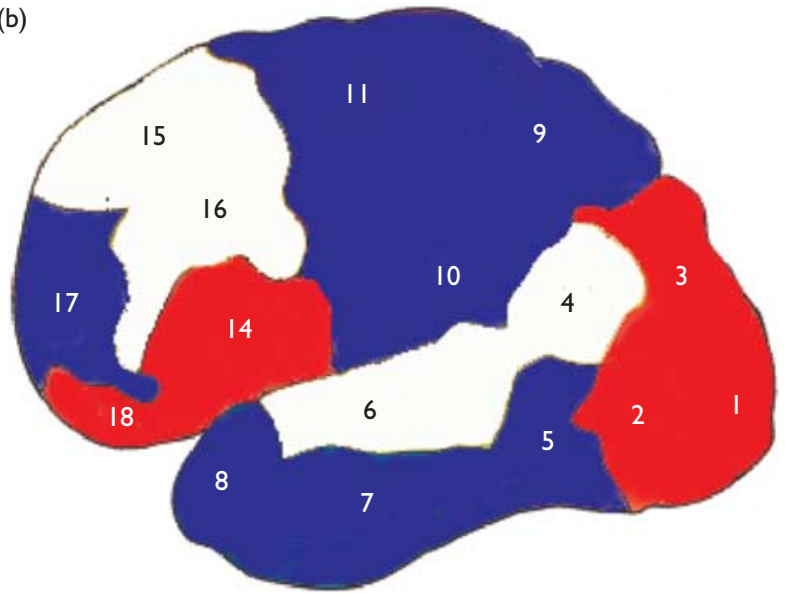

Spatial principal component analysis (sPCA) results. (a) The two components as yielded by the sPCA analysis of the electrical neuroimaging data comparing the own-body transformation task with the mirror task and lateralization task [sPCA1 (intrinsic): blue; sPCA2 (extrinsic): red]. Note the contradictive pattern between the two components. (b) The intrinsic and extrinsic systems are shown. The first system was composed mostly of the functional Brodmann Areas (fBAs) $5,7-11,17$ (blue), and the second component of the fBAs $1,2,3,14,18$ (red). Common regions $(4,6,15,16)$ are shown in white.

regions; fBAs 14,18) systems. This is concordant with a study by Gusnard et al. [19] showing an increase in brain activity at fBA 17 (intrinsic system) and decrease in brain activity at fBA 18 (extrinsic system) during self-related tasks, suggesting that fBA 17 might be associated with selfreferential processing [3,19]. Mesulam [20] also highlighted the role of this region in transposing one's 'reference point from self to other, from here to there, and from now to then', compatible with the present findings. fBA 17 is also interconnected with limbic structures, suggesting a role for this region in the integration of stimuli regarding the 'internal milieu' and emotional processing $[3,18,19]$. This frontal activity also comprises specialized attention-regions as a part of a frontoparietal network overlapping with the 'default network', which is hypothesized to be crucial for relating perceived information to self-related information [6].
With respect to the frontal extrinsic system, fBA 14 comprises Broca's area, a major language region, and fBA18 (and fBA 16) are involved in processing of external stimuli through 'working memory' [21]. In addition, fBA 18 , has been implicated in executive control and has a high density of interconnections with other high-order areas in the cerebral cortex, and through them it might activate or suppress different networks [22]. Therefore, although considered to be a self-related region, we speculate that this region deals mainly with the external environment through anticipation of the coming stimuli, and this in turn might explain its inclusion in the extrinsic system. These data should be regarded with caution as activation at the frontal cortex shows variability among studies and also among individuals in some studies [9]. Visual stimuli and paradigms, as used in these studies, may have targeted primarily more posterior brain regions, making the classification of the prefrontal regions to 'extrinsic' and 'intrinsic,' based on these visual stimuli, less reliable. Further research is therefore needed for defining prefrontal involvement in the intrinsic and extrinsic systems.

\section{Intrinsic and extrinsic brain regions in the posterior cortex}

With respect to the posterior cortex, our analysis showed two key regions involving the intrinsic system: the lateral temporal (fBA 5) and the temporo-parietal (fBA 10) cortex. These regions have been shown to play a key role in the mental imagery of one's own body [12,13] and other self-related processes such as agency or visuospatial perspective taking [10], and have been considered crucial for 'self-projection' [11].

The posterior component of the extrinsic system, as found here, is composed mostly of primary and secondary visual sensory cortices, responding to the presentation of external visual stimuli [18,23,24]. These processes are not task-specific but characterize the acquisition and initial processing of different external inputs, supplying information that is necessary for the organism to behave in the environment $[18,19]$. The extrinsic system, as found here, is generally compatible with the classical topography of the cortical visual system in the posterior brain, including the two cortical visual 'what' and 'where' systems, each beginning in the primary visual cortex, diverging within the prestriate cortex and then coursing either ventrally into the temporal cortex or dorsally into the parietal cortex [24,25]. Subsequent links of the regions in the 'what' system with the brain regions of the intrinsic system have been described [25].

\section{Right hemispheric dominance of the intrinsic system for self-projection}

Right and left hemispheres were found to code differentially for self-projection. This is suggested by the higher activity of the 'intrinsic' sPCA1 for OBT than for 
Fig. 3

(a)
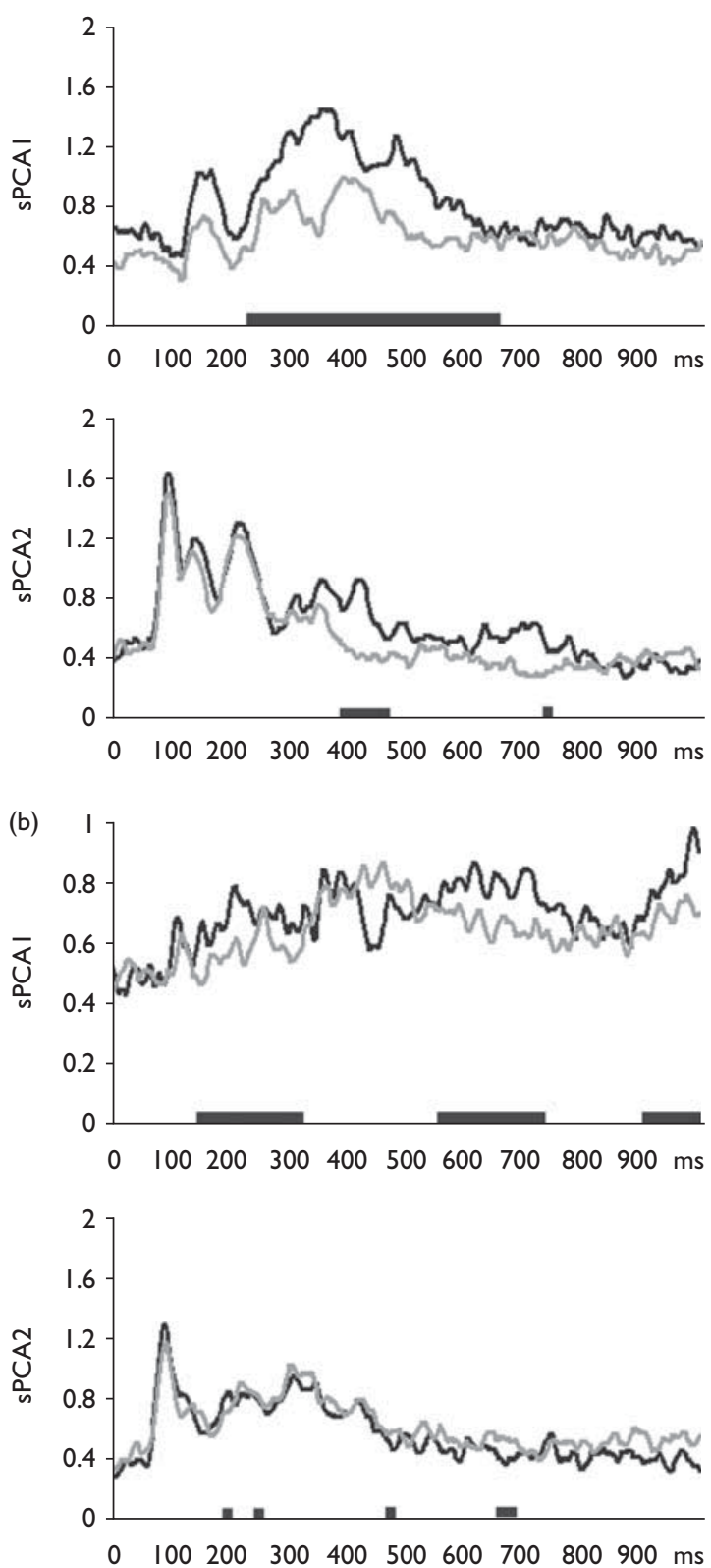

Left hemisphere
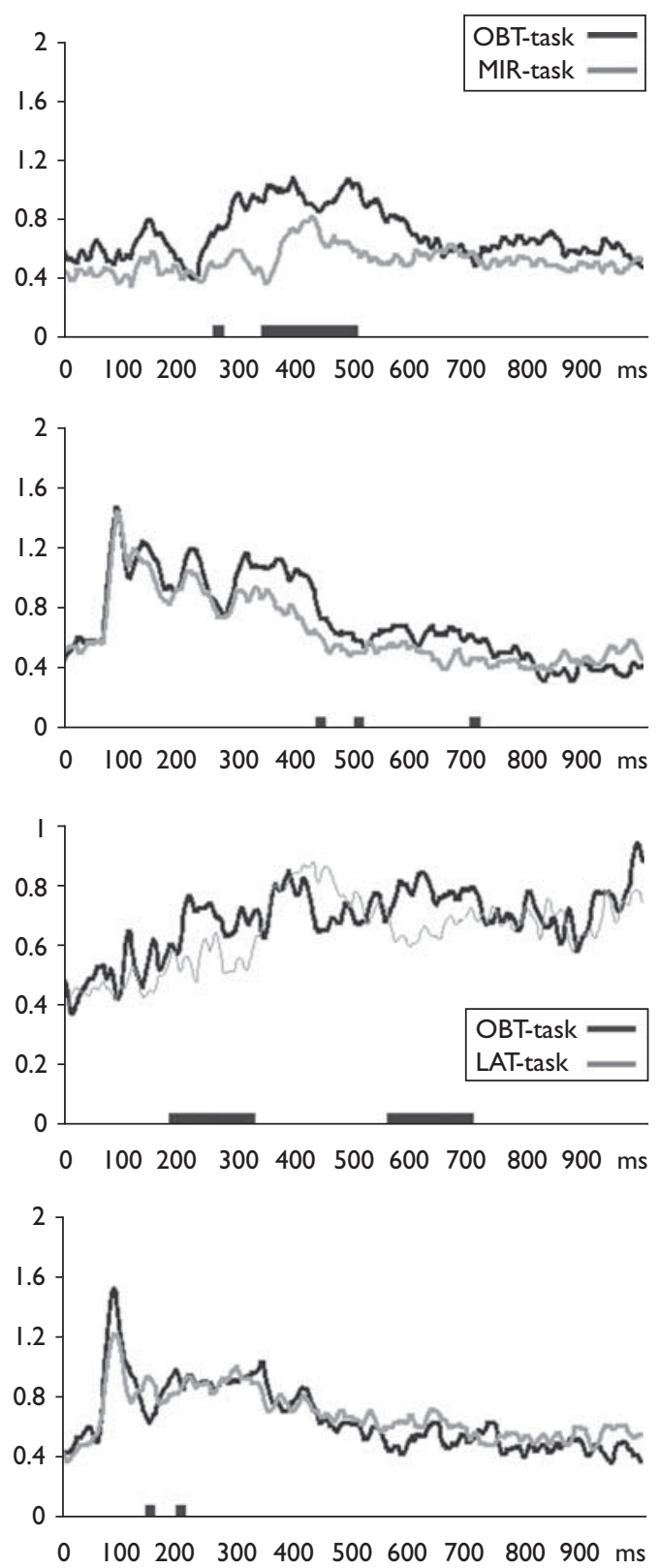

Time course of principal component analysis (PCA) factor loadings. Results of the PCA (factor loadings) showing the two distinct components over time for each hemisphere. Significant differences are shown by the black bars. (a) Comparison of the own-body transformation (OBT) (black) and mirror (MIR) (grey) tasks. Note the difference between the two tasks for the first component from approximately 250 to $650 \mathrm{~ms}$ in the right hemisphere and from approximately 350 to $500 \mathrm{~ms}$ in the left hemisphere. (b) Comparison of OBT (black) and lateralization (LAT) (grey) tasks. Note the difference between the two tasks for the first component from approximately 200 to 350 and approximately 600 to 700 ms in both the hemispheres.

MIR and LAT-tasks at around $300-600 \mathrm{~ms}$, and for the MIR-task than the LAT-task in a similar time-window. This right hemispheric dominance is concordant with earlier studies, using various self-related and body-related paradigms including visuospatial perspective taking and own-body mental imagery [10,12]. sPCA2 did not show major differences between the hemispheres, con- cordant with its presumable role in external stimulus perception.

\section{Timing of activation for the intrinsic and extrinsic systems}

This study, using electroencephalogram with high temporal-resolution, also allowed us to specify a time-window 
between 300 and $400 \mathrm{~ms}$ in which sPCA1 was peaking for the OBT and MIR tasks, whereas sPCA2 was peaking for all tasks around $100 \mathrm{~ms}$. These results are consistent with earlier studies [12,13], which used the same tasks (OBT, MIR, and LAT) but different analysis methods (evoked potential mapping and LAURA source localization), showing occipital and frontal activations at approximately $0-250 \mathrm{~ms}$ after stimulus presentation peaking at approximately $100 \mathrm{~ms}$, and lateral temporal and parietal activations from approximately 250 to $500 \mathrm{~ms}$ related to task performance. These temporal differences support the differentiation between the intrinsic and extrinsic systems, as extrinsic perception generally precedes intrinsic high-order processing [1] by approximately $200 \mathrm{~ms}$.

In conclusion, this study suggests that the cerebral cortex may be functionally divided into two main systems: intrinsic and extrinsic. The extrinsic system is related to perception of external stimuli, whereas the intrinsic system refers to the 'internal milieu'. 'Self-projection' is proposed here to take a major role in the activity of the intrinsic system, from one's actual self-location within the body to probable future imaginary self-locations.

\section{Acknowledgement}

The authors thank Dr Lars Schwabe for helpful comments on the manuscript.

\section{References}

1 Mesulam MM. Principles of behavioral and cognitive neurology. Oxford: Oxford University Press; 2001.

2 Raichle ME, Mintun MA. Brain work and brain imaging. Annu Rev Neurosci 2006; 29:449-476.

3 Raichle ME, Snyder AZ. A default mode of brain function: a brief history of an evolving idea. Neurolmage 2007; 37:1083-1090. Discussion 1097-1099.

4 Raichle ME, MacLeod AM, Snyder AZ, Powers WJ, Gusnard DA, Shulman GL. A default mode of brain function. Proc Natl Acad Sci USA 2001; 98:676-682.

5 Mantini D, Perrucci MG, Del Gratta C, Romani GL, Corbetta M. Electrophysiological signatures of resting state networks in the human brain. Proc Natl Acad Sci USA 2007; 104:13170-13175.

6 Fox MD, Snyder AZ, Vincent JL, Corbetta M, Van Essen DC, Raichle ME. The human brain is intrinsically organized into dynamic, anticorrelated functional networks. Proc Natl Acad Sci USA 2005; 102:9673-9678.
7 Cabeza R, Nyberg L. Imaging cognition II: an empirical review of 275 PET and fMRI studies. J Cogn Neurosci 2000; 12:1-47.

8 Golland $\mathrm{Y}$, Bentin S, Gelbard H, Benjamini Y, Heller R, Nir Y, et al. Extrinsic and intrinsic systems in the posterior cortex of the human brain revealed during natural sensory stimulation. Cereb Cortex 2007; 17:766-777.

9 Golland Y, Golland P, Bentin S, Malach R. Data-driven clustering reveals a fundamental subdivision of the human cortex into two global systems. Neuropsychologia 2008; 46:540-553.

10 Blanke O, Arzy S. The out-of-body experience: disturbed self-processing at the temporo-parietal junction. Neuroscientist 2005; 11:16-24.

11 Buckner RL, Carroll DC. Self-projection and the brain. Trends Cogn Sci 2007; 11:49-57.

12 Arzy S, Thut G, Mohr C, Michel CM, Blanke O. Neural basis of embodiment: distinct contributions of temporoparietal junction and extrastriate body area. J Neurosci 2006; 26:8074-8081.

13 Blanke O, Mohr C, Michel CM, Pascual-Leone A, Brugger P, Seeck M, et al. Linking out-of-body experience and self processing to mental own-body imagery at the temporoparietal junction. $J$ Neurosci 2005 25:550-557.

14 Arzy S, Seeck M, Ortigue S, Spinelli L, Blanke O. Induction of an illusory shadow person. Nature 2006; 443:287.

15 Blanke O, Landis T, Spinelli L, Seeck M. Out-of-body experience and autoscopy of neurological origin. Brain 2004; 127:243-258.

16 Desikan RS, Segonne F, Fischl B, Quinn BT, Dickerson BC, Blacker D, et al. An automated labeling system for subdividing the human cerebral cortex on MRI scans into gyral based regions of interest. Neurolmage 2006; 31:968-980.

17 Pascual-Marqui RD, Michel CM, Lehmann D. Low resolution electromagnetic tomography: a new method for localizing electrical activity in the brain. Int J Psychophysiol 1994; 18:49-65.

18 Shulman GL, Corbetta M, Buckner RL, Raichle ME, Fiez JA, Miezin FM, et al. Top-down modulation of early sensory cortex. Cereb Cortex 1997; 7:193-206.

19 Gusnard DA, Akbudak E, Shulman GL, Raichle ME. Medial prefrontal cortex and self-referential mental activity: relation to a default mode of brain function. Proc Natl Acad Sci USA 2001; 98:4259-4264.

20 Mesulam MM. The human frontal lobes: transcending the default mode through contingent encoding. In: Stuss DT, Knight RT, editors. Principles of frontal lobe function. Oxford: Oxford University Press; 2002. pp. 8-30.

21 Baddeley A. Working memory. Science (New York) 1992; 255:556-559.

22 Rao SC, Rainer G, Miller EK. Integration of what and where in the primate prefrontal cortex. Science (New York) 1997; 276:821-824.

23 Grill-Spector K, Malach R. The human visual cortex. Annu Rev Neurosci 2004; 27:649-677.

24 Ungerleider LG, Mishkin M. Two cortical visual systems. In: Ingle D, Goodale M, Mansfield R, editors. Analysis of visual behavior. Cambridge, Massachusetts: MIT Press; 1982. pp. 549-586.

25 Mishkin M, Unterleider LG, Macko KA. Object vision and spatial vision: two cortical pathways. In: Bechtel W, Mandik P, Mundale J, Stufflebeam RS, editors. Philosophy and the neurosciences: a reader. Oxford: Basil Blackwell; 2001. pp. 199-208. 